\title{
Kesintisiz Güç Kaynağı Çıkış Gücü Düzenlemesi için Bulanık Mantık ve Kazanç Çizelgesi Uyarlanır Tabanlı PI Kontrolörlerin Performans Karşılaştırılması
}

\author{
Vedat Gül ${ }^{1 *}$, Savaş Şahin ${ }^{2}$ \\ 1* Tescom Elektronik A.Ş, Department of Research and Development, İzmir, Türkiye, (ORCID: 0000-0003-3980-2118), vedatgul2013@gmail.com \\ ${ }^{2}$ Izmir Kâtip Çelebi University, Faculty of Engineering and Architecture, Department of Electrical and Electronics Engineering, İzmir, Türkiye (ORCID: 0000- \\ 0002-4165-3441), sahin.savas@yahoo.com
}

(2nd International Conference on Access to Recent Advances in Engineering and Digitalization (ARACONF)-10-12 March 2021)

(DOI: 10.31590/ejosat.903268)

ATIF/REFERENCE: Gul V, Sahin S, Kesintisiz Güç Kaynağı Çıkış Gücü Düzenlemesi için Bulanık Mantık ve Kazanç Çizelgesi Uyarlanır Tabanlı PI Kontrolörlerin Performans Karşılaştırılması, Mart 2021, Avrupa Bilim ve Teknoloji Dergisi, (24), 416-420.

$\ddot{\mathbf{O z}}$

Bu çalışmada, kesintisiz güç kaynağı (KGK) çıkış gücü düzenlemesi için geleneksel oransal-integral (PI) kontrolör, bulanık mantık (BM) ve kazanç çizelgesi uyarlanır (KÇU) tabanlı PI kontrolörlerin tasarımları ve performansları sunulmaktadır. KGK'larda evirici için kontrolör tasarımında yaygın olarak sabit katsayılı geleneksel PI kontrolör kullanılmaktadır. Ancak sabit katsayılı kontrolör yerine katsayıları güncellenen kontrolörler daha iyi performans sağlayabilirler. Bu nedenle, evirici çıkış gücü düzenlemesi için kontrolör katsayılarını güncelleyen, BM ve KÇU tabanlı PI kontrolör tasarımları gerçekleştirilmiştir. Benzetim ve deneysel çalışmalarda, $5 k W$ KGK sisteminin performans sonuçları sistem çıkış geriliminin toplam harmonik bozulma ve ortalama mutlak hata değerleri açısından karşılaştırılmıştır. Deneysel çalışma sonuçlarına göre, en iyi sonucu KÇU tabanlı PI kontrolör sağlamıştır.

Anahtar Kelimeler: Kesintisiz güç kaynağı, Bulanık mantık, Kazanç çizelgesi uyarlanır, PI kontrolör

\section{Performance Comparison of Fuzzy Logic and Gain Scheduling Adaptive Based PI Controllers for Uninterruptible Power Supply Output Power Regulation}

\begin{abstract}
In this study, the designs of the conventional proportional-integral (PI) controller, fuzzy logic (FL), and gain scheduling adaptive (GSA) based PI controllers and their performances are presented for uninterruptible power supply (UPS) output power regulation. The conventional PI controller having constant parameters is commonly used for the inverter in UPS. However, controllers whose parameters are updated instead of using fixed parameters one might provide better performance. Hence, FL and GSA based PI controller designs, which update the controller parameters for the inverter output power regulation, have been implemented. In simulation and experimental studies, the performance results of the $5 \mathrm{~kW}$ UPS system have been compared in terms of total harmonic distortion and mean absolute error values of the system output voltage. According to the obtained results of the experimental study, the best result was obtained by the GSA based PI controller.
\end{abstract}

Keywords: Uninterruptible power supply, Fuzzy logic, Gain scheduling adaptive, PI controller. 


\section{Giriş}

Güç sistemlerinin önemli bir parçası olan kesintisiz güç kaynakları (KGK) elektrik şebekesinin kesildiği veya düzensiz olduğu yerlerde kullanılır ([1], Rahman ve ark [2]). KGK sisteminde arzu edilen güç düzenlemesi ve düşük toplam harmonik bozulma (THB) için gerilim düzenlemeli evirici tasarımı geliştirilmiştir (Yeh ve Manjrekar [3]). Eviricilerde çıkış gücü düzenlemesinin geleneksel oransal-integral (PI) kontrol ile yapıldığı birçok çalışma mevcuttur ancak sabit katsayılı PI kontrolörün değişken yüklere karşı sistem performansı kısıtlıdır [4-6]. Daha iyi sistem performansı elde etmek için kontrolör parametreleri güncellenebilen bulanık mantık (BM) tabanlı kontrolörler geliştirilmiştir ([7], Mir ve ark. [8], Jian ve ark. [9], Ding ve ark. [10], Thao ve ark. [11], Li ve ark. [12]). Sayısal işaret işlemci tabanlı PID kontrolör ile eviricinin doğrusal olmayan yüklere karşı performansı dinamik olarak güncellenen BM yöntemi ile bütünleştirilmiştir (Jian ve ark. [9]). $20 \mathrm{~kW}$ bir evirici BM tabanlı kontrolörle gerçekleştirmişlerdir (Ding ve ark. [10]). Elektrik şebekesine bağlı fotovoltaik bir evirici için BM tabanlı kontrolörle başarılı sonuçlar elde edilmiştir (Thao ve ark. [11]). Bir başka çalışmada eviricinin BM tabanlı PID kontrolör yöntemi performansının daha iyi olduğu yayımlanmıştır (Li ve ark. [12]). Diğer yandan, uyarlanır kontrolör ile yapılan evirici tasarımı çalışmaları da bulunmaktadır (Chen ve ark. [13], Jiao ve ark. [14], Yılmaz ve ark. [15]). Tasarlanan bir evirici için çeşitli çalışma yüklerine karşılık gelen kontrol kazanç katsayıları hesaplanmış ve kullanılmıştır (Chen ve ark. [13]). Şebekeye bağlı tek fazlı eviricinin şebeke empedans değişikliklerine karşı sistemi gürbüz yapabilmek için kontrolör parametrelerini ayarlayabilen kazanç çizelgelemeli uyarlanır (KÇU) kontrolör geliştirmişlerdir (Jiao ve ark. [14]). Bir eviricinin kontrolünde geleneksel PID yerine KÇU tabanlı PI kontrolör kullanımının daha uygun olduğu gösterilmiştir (Yılmaz ve ark. [15]).

Bu çalışmada, tasarlanan $5 \mathrm{~kW}$ 'lık bir KGK tek fazlı yarım köprü evirici devresinin çıkış gerilimi düzenlemesi için geleneksel PI, BM ve KÇU tabanlı PI kontrolörler tasarlanmıştır. Çıkış gücü düzenlemesi performansları THB ve ortalama mutlak hata (MAE) açısından karşılaştırılmıştır. Benzetim çalışmaları MATLAB/Simulink ortamında ve gerçek sistem testleri deney düzeneği üzerinde yapılmıştır. Geliştirilen kontrolörlerden elde edilen sonuçlara göre tam yükte KÇU tabanlı PI kontrolör performansının diğer kontrolör yöntemlerine göre daha iyi olduğu gözlenmiştir. Çıkış gerilimi THB ve MAE değerleri sırasıyla $\% 0.9$ ve 2.7047 olarak elde edilmiştir.

Çalışmanın geriye kalan bölümleri: Bölüm 2'de KGK evirici çıkış devresi tasarımı, kontrol yöntemleri ve çıkış gerilimi düzenlemesi için tasarlanan geleneksel PI, BMK ve KÇU tabanlı PI kontrolör algoritmaları sunulmuştur. Bölüm 3 'te benzetim çalışmaları ve gerçeklenen deney sonuçları verilmiştir. Bölüm 4'te sonuçlar ve olası gelecek çalışmalar açıklanmıştır.

\section{Materyal ve Metot}

\subsection{Tasarlanan KGK Evirici}

$5 \mathrm{~kW}$ KGK tasarımı tek fazlı yarım dalga köprü evirici, dengeleyeci, akım ve gerilim algılayıcılar ve mikrokontrolör tabanlı anakart kısımlarımdan oluşmaktadır (Şekil 1). Evirici tasarımında, $12868 \mathrm{~Hz}$ frekansla uyumlu anahtarlama elemanları olarak yalıtılmış iki kapılı transistör kullanılmıştır. Tasarlanan eviricide güç kontrolü gerilim ve akım değişkenlerinin algılayıcılar aracılığı ile yapılır. Ölçülen akım ve gerilim değişkenleri dsPIC akım ve gerilim kontrol algoritması içerisinde geri-bildirim olarak kullanılır. Tasarımda sayısal işaret işlemcisi olarak dsPIC33EP512GM710 kullanılmıştır (Şekil 2) [16].

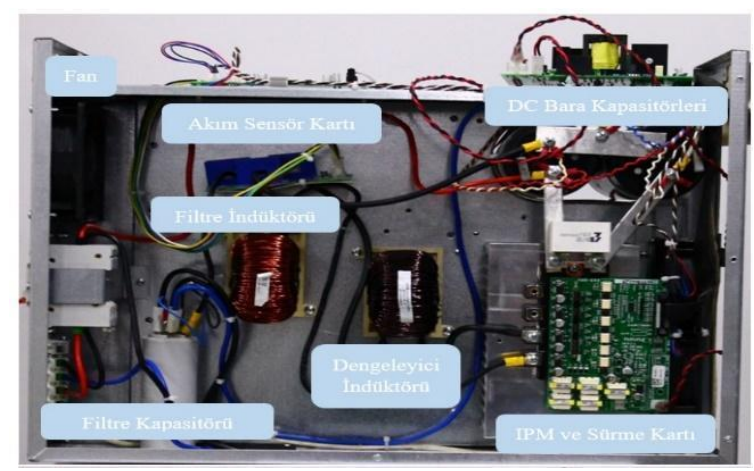

Şekil 1. Tasarlanan 5kW KGK donanımı

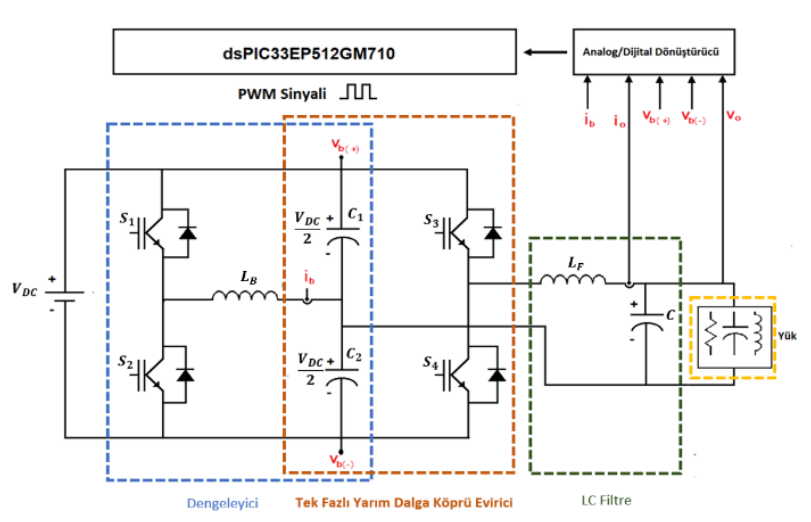

Şekil 2. Tasarlanan evirici devresi

Eviricide kullanılan geleneksel PI kontrolör $u(t)=$ $K_{p} e(t)+K_{i} \int_{0}^{t} e(t) d(t)$ ait kazanç parametreleri gerçek sistem üzerinden Ziegler-Nichols titreşim yöntemi ile bulunmuştur [17]. Sistem için referans gerilim değeri $220 V_{R M S}$ olarak alınmıştır. Elde edilen kontrolör parametreleri $K_{p}=50$ ve $K_{i}=30$ olarak bulunmuştur. Evirici için tasarlanan BM tabanlı PI kontrolör yapısı Şekil 3'de verilmiştir. İç döngüde akım kontrolü ayrı bir PI kontrolör ile yapılmış, gerçek çıkış akımı $I_{k}^{o}$ referans akım ise $22 A$ 'dir. İç döngüde kullanılan akım kontrolü için PI kontrolör parametreleri titreşim yöntemine göre $K_{p}=5000$ ve $K_{i}=400$ olarak bulunmuştur. Evirici dış döngüde çıkış gerilim düzenlemesi için kullanılan BM tabanlı PI kontrolörde gerçek çıkış $V_{k}^{o}$ olarak alınmıştır. BM girişinde çıkış gerilim $V_{k}^{o}$ ve arzu edilen çıkış gerilim $V_{k}^{r e f}$ farkı ve farklar arasındaki değişim dikkate alınmıştır. 


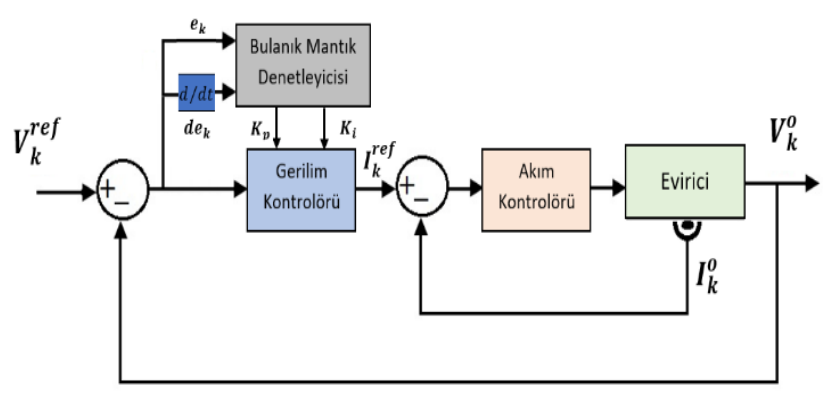

Şekil 3. Çıkış gerilimi düzenlemesi için BM tabanlı PI kontrolör

Hata, hatanın değişimi, BM çıkış değerleri $K_{p}$ ve $K_{i}$ için üçgen üyelik fonksiyonları kullanılmıştır. $K_{p}$ ve $K_{i}$ katsayılarına ait kural tablosu Tablo 1'de uzman görüşü ve deneme yanılma ile oluşturulur. Burada sıfir (S), negatif büyük (NB), negatif orta $(\mathrm{NO})$, pozitif büyük $(\mathrm{PB})$, pozitif orta $(\mathrm{PO})$, küçük $(\mathrm{K})$, küçük orta $(\mathrm{KO})$, orta $(\mathrm{O})$, büyük orta (BO) ve büyük (B) olarak belirlenmiştir [18] [19]. Tasarlanan BM'de $e$ ve de giriş değerleri $((-220)-(+220)), K_{p}$ ve $K_{i}$ çıkış değerleri ise sırasıyla (40-60) ve (20-40) olarak alınmıştır. Mamdani çıkarım yöntemine göre en küçük operatör ve durulaştırma için ağırlıklı ortalama kullanılmıştır.

Tablo 1. $K_{p}$ ve $K_{i}$ için kural tablosu

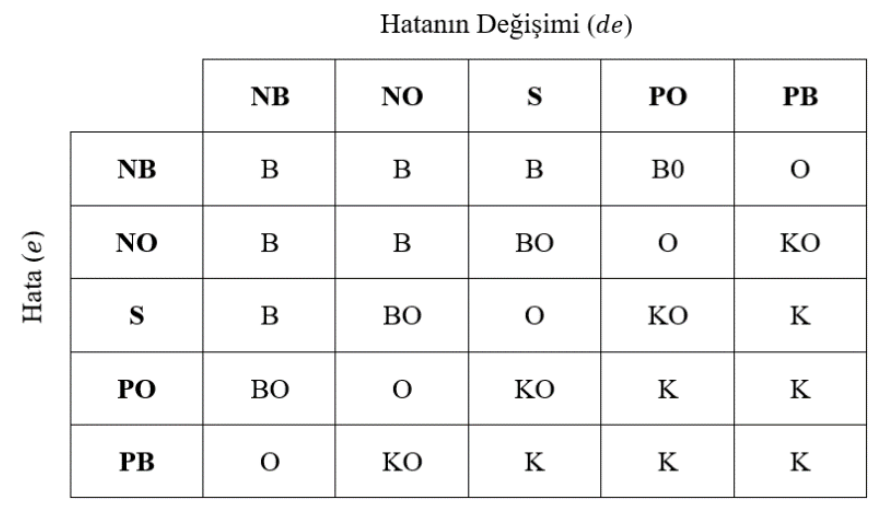

KÇU tabanlı PI kontrolör tasarımı Şekil 4'te verilmiş, eviricinin farklı çalışma koşullarında uygun parametre seçimleri evirici çıkış gücü kontrolör katsayıları için yapılmıştır (Tablo 2). $\mathrm{Bu}$ tabloda verilen yük değerleri evirici çıkış gerilimi THB değerleri ölçülerek belirlenmiştir.

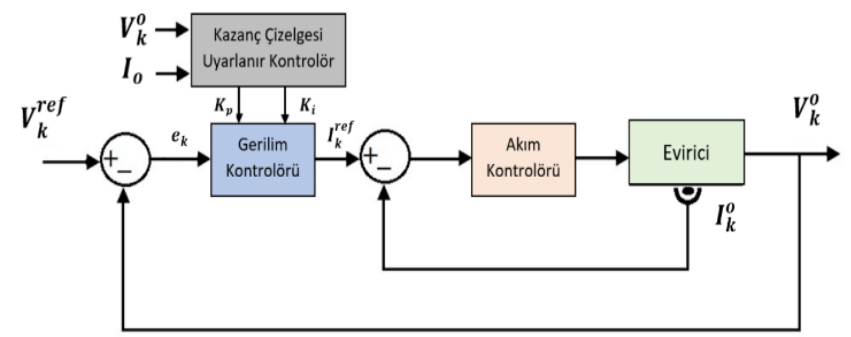

Şekil 4. Çıkış gerilimi düzenlemesi için KÇU tabanlı PI kontrolör
Tablo 2. KÇU tabanlı PI kontrolör katsayıları

\begin{tabular}{|c|c|c|c|}
\hline Yük oranı (\%) & Güç $(W)$ & $\boldsymbol{K}_{\boldsymbol{p}}$ & $\boldsymbol{K}_{\boldsymbol{i}}$ \\
\hline 20 & 1000 & 44 & 36 \\
\hline 40 & 2000 & 48 & 32 \\
\hline 60 & 3000 & 52 & 28 \\
\hline 80 & 4000 & 56 & 24 \\
\hline 100 & 5000 & 60 & 20 \\
\hline
\end{tabular}

\section{Araştırma Sonuçları ve Tartışma}

\subsection{Benzetim Çalışmaları}

Benzetim çalışmaları MATLAB/Simulink ortamında tasarlanan evirici ile yapılmıştır. Geleneksel PI kontrolör yöntemi test edilmiştir (Şekil 5). Referans sinyali $\left(220 V_{R M S} / 50 \mathrm{~Hz}\right)$ sinüs biçiminde seçilmiştir. Ayrıca sinüzoidal PWM uygulamak için taşıyıcı üçgen sinyal kullanılmış ve örnekleme frekansı $f_{s}=$ $12868 \mathrm{~Hz}$ olarak belirlenmiştir. Önceki bölümde açıklandığ 1 gibi PI kontrolör parametreleri $K_{p}=50$ ve $K_{i}=30$ olarak alınmıştır.

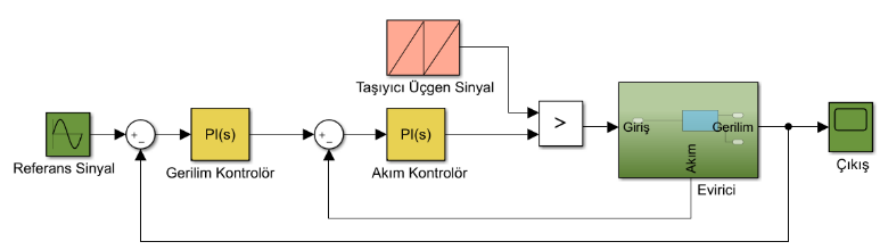

Şekil 5. Geleneksel PI kontrolör simulink tasarımı

BM tabanlı PI kontrolör benzetim çalışmaları Şekil 6'daki gibi yapılmıştır. Evirici çıkış gerilimi ve referans gerilim farkı ile hata değeri elde edilir. Hata ve hatanın değişimi BM aracında giriş değerleri olarak kullanılır. BM işlemleri sonucunda $K_{p}$ ve $K_{i}$ katsayıları elde edilmiştir.

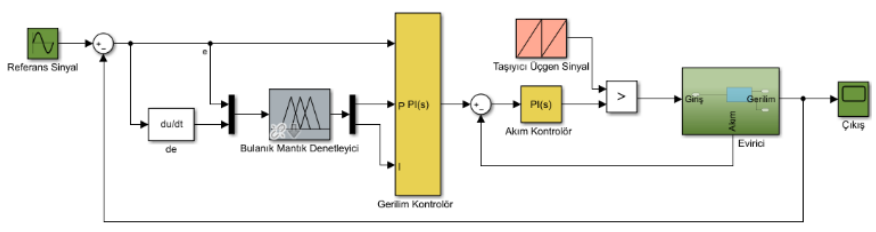

\section{Şekil 6. BM tabanlı PI kontrolör tasarımının simulink modeli}

KÇU tabanlı PI kontrolör için Tablo 2'de elde edilen parametre değerleri ile $K_{p}$ ve $K_{i}$ kazanç çizelgesi kullanılmıştır. Bu tablo ile $K_{p}$ ve $K_{i}$ kazanç katsayıları ve kapalı döngü sistemi hata değeri ile PI kontrolör çıkışı eviriciye uygulanır (Şekil 7).

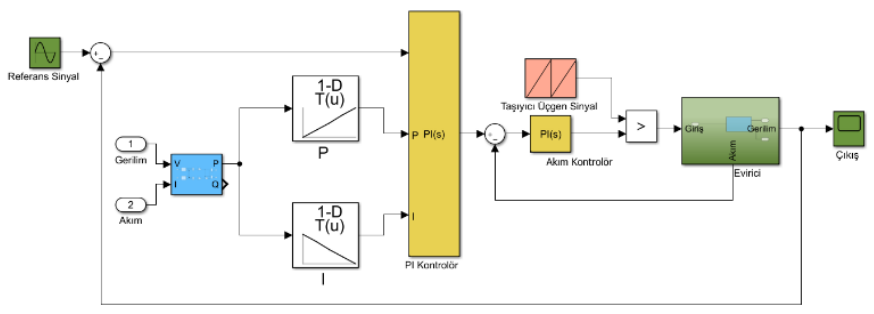

Şekil 7. KÇU tabanlı PI kontrolörün simulink modeli 
Benzetim çalışmaları sonucunda elde edilen zaman düzlemi çıkış gerilimi ve çıkış akımı değerleri birbiri ile aynıdır, Şekil 8'de sunulmuştur. Simulink benzetim ortamında tasarlanan evirici üzerinde geleneksel $\mathrm{PI}, \mathrm{BM}$ ve KÇU tabanlı PI kontrolör performansları karşılaştırılmıştır (Tablo 3). Rezistif tam yükte (\%100) yapılan performans testleri sonucunda KÇU tabanlı PI kontrolör yöntemi çıkış gerilim THB ve MAE değerleri sırasıyla $\% 0.60$ ve 1.6698 olarak elde edilmiştir. Çıkış gerilim THB ve MAE sırasıyla Denklem 1 ve 2'de verilmiştir. Burada $V_{h}$ çıkış gerilimini $e_{k}$ kapalı döngü kontrol sistemi hatasını gösterir.

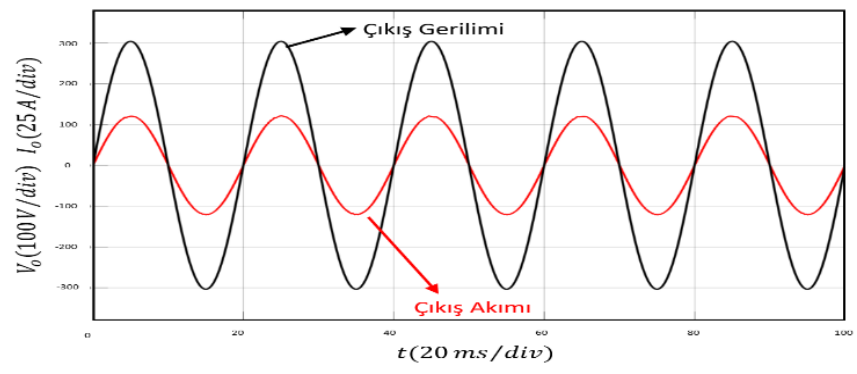

Şekil 8. Çıkış gerilimi ve akımı

Tablo 3. Benzetim ortamında kontrolör performansları

\begin{tabular}{|c|c|c|}
\hline & \multicolumn{2}{|c|}{ (Rezistif Yük 100\%) } \\
\hline Kontrolör & THB (\%) & MAE \\
\hline Geleneksel PI & 1.05 & 1.7121 \\
\hline BM tabanlı PI & 0.75 & 1.6805 \\
\hline KÇU tabanlı PI & 0.60 & 1.6698 \\
\hline
\end{tabular}

$T H B=\frac{\sqrt{\sum_{h=2}^{\infty} \quad V_{h}^{2}}}{V_{1}}$

$$
M A E=\frac{1}{n} \sum_{k=1}^{n} \quad\left|e_{k}\right|
$$

\subsection{Deneysel Çalışmalar}

Deney düzeneği için dsPIC33EP512GM710 işlemcinin kullanıldığ 1 anakart, evirici devresi, akım ve gerilim algılayıc1 devreler, evirici devresi üzerinde kullanılan akıllı güç modülü ve sürücü kartı tasarlanmıştır (Şekil 9).

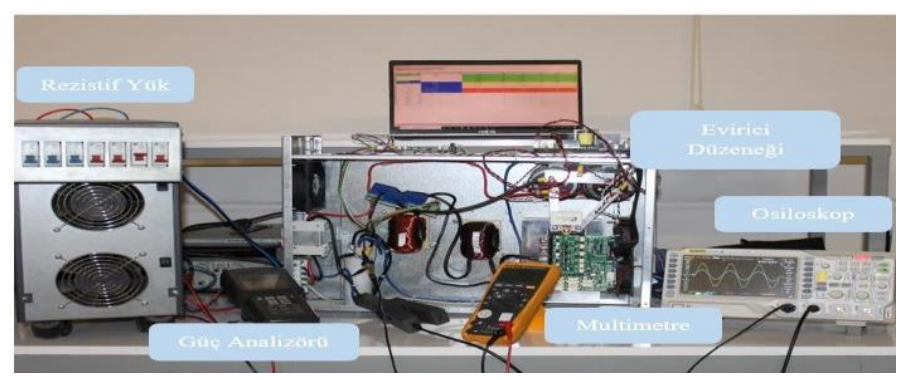

Şekil 9. Deney düzeneği tasarımı
Gerçek sistem çalışmaları tasarlanan evirici ile yapılmıştır. Geleneksel PI kontrolör yöntemi test edilmiştir (Şekil 10). Referans sinyali $V_{k}^{r e f}=220 V_{R M S}$ sinüs biçiminde seçilmiştir. Ayrıca dijital olarak sinüzoidal PWM tekniğini uygulayabilmek için örnekleme frekansı $f_{S}=12868 \mathrm{~Hz}$ ve bara gerilimi $360 V_{A C}$ olarak belirlenmiş̧tir. PI kontrolör parametreleri $K_{p}=50$ ve $K_{i}=$ 30 olarak alınmıştır.

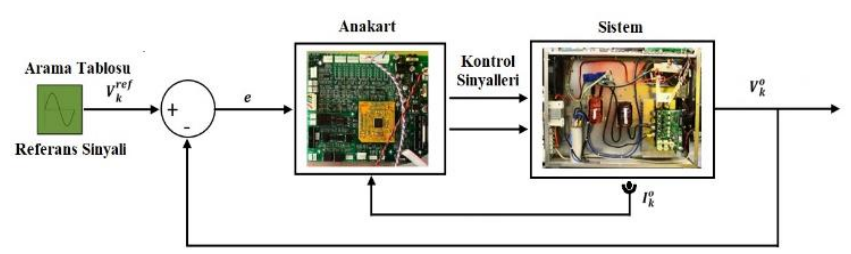

Şekil 10. Geleneksel PI kontrolör deney düzeneği tasarımı

BM tabanlı PI kontrolör Şekil 11'deki gibi gerçeklenmiştir. Evirici çıkış gerilimi ve referans gerilim farkı ile hata değeri elde edilir. Hata ve hatanın değişımi BM aracında giriş değerleri olarak kullanılır. BM işlemleri sonucunda $K_{p}$ ve $K_{i}$ katsayıları elde edilmiştir.

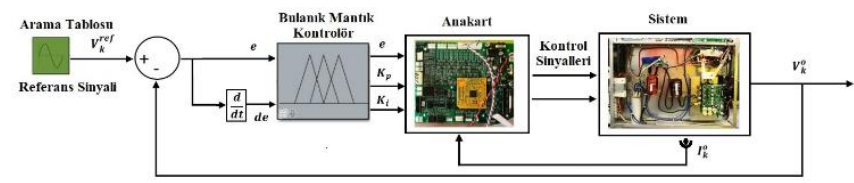

Şekil 11. BM tabanlı PI kontrolör deney düzeneği tasarımı

KÇU tabanlı PI kontrolör için Tablo 2'de elde edilen parametre değerleri ile $K_{p}$ ve $K_{i}$ kazanç çizelgesi kullanılmıştır. Bu tablo ile $K_{p}$ ve $K_{i}$ kazanç katsayıları ve kapalı döngü sistemi hata değeri ile PI kontrolör çıkışı eviriciye uygulanır (Şekil 12).

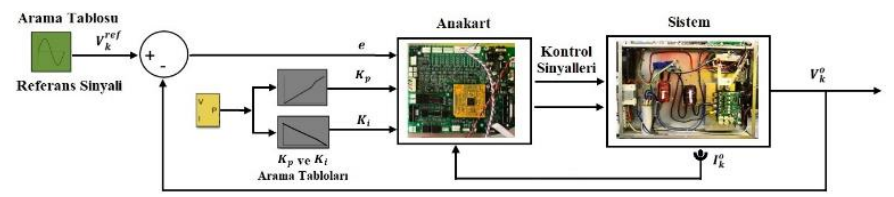

Şekil 12. KÇU tabanlı PI kontrolör deney düzeneği tasarımı

Gerçek sistem testleri sonucunda elde edilen zaman düzlemi çıkış gerilimi ve çıkış akımı değerleri neredeyse aynıdır, Şekil 13 'de sunulmuştur. Tasarlanan evirici üzerinde geleneksel PI, BM ve KÇU tabanlı PI kontrolör performansları karşılaştırılmıştır (Tablo 4). Rezistif tam yükte (\%100) yapılan performans testleri sonucunda KÇU tabanlı PI kontrolör yöntemi çıkış gerilim THB ve MAE değerleri sırasıyla \%0.9 ve 2.7047 olarak elde edilmiştir.

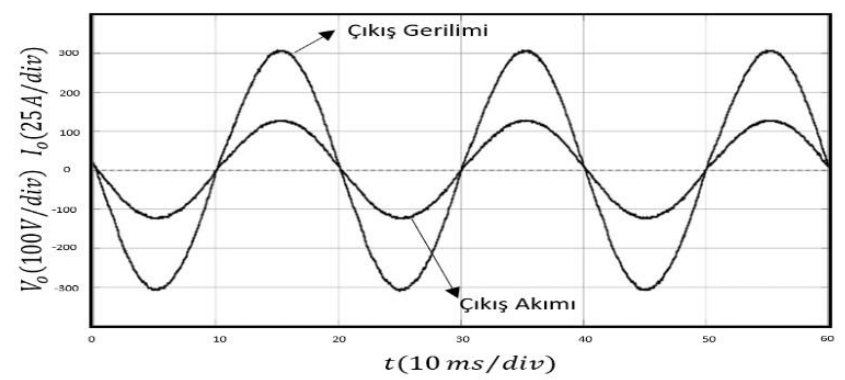

Şekil 13. Çıkış gerilimi ve akımı 
Tablo 4. Deney düzeneğinde kontrolör performanslart

\begin{tabular}{|c|c|c|}
\hline & \multicolumn{2}{|c|}{ (Rezistif Yük 100\%) } \\
\hline Kontrolör & THB (\%) & MAE \\
\hline Geleneksel PI & 1.7 & 3.0719 \\
\hline BM tabanlı PI & 1.2 & 2.7888 \\
\hline KÇU tabanlı PI & 0.9 & 2.7047 \\
\hline
\end{tabular}

\section{Sonuç}

$\mathrm{Bu}$ çalışma ile tasarlanan $5 \mathrm{~kW}$ KGK ve evirici için tasarlanmış geleneksel PI, BM ve KÇU tabanlı PI kontrolör algoritmalarının benzetim ve gerçek sistem sonuçları sunulmaktadır. Yapılan testler sonucunda tam yükte KÇU tabanlı PI kontrolör performansının diğerlerine göre daha iyi sonuç verdiği gözlenmiştir. Çıkış gerilim THB ve MAE değerleri tam yük altında karşılaştırılmıştır. İleriye dönük çalışmalarda değişken ve doğrusal olmayan yük altında kontrolör yöntemleri denenebilir ve başarılı sonuçlar elde edilebilir.

\section{Teşekkür}

Laboratuvar ve deney düzeneği kurulumu için sağladığı imkanlardan dolayı TESCOM A.Ş.' ye teşekkür ederiz.

\section{Kaynakça}

1. Deng H, Omganti R, Srinivasan D. Digital control of singlephase UPS inverters with modified PWM technique. IEEE 35th Annual Power Electronics Specialists Conference. 2004 June; 1365-1371.

2. Rahman MA, Quaicoe JE, Esmail AR, Choudhury MA. Delta Modulated Rectifier-Inverter for Uniterruptible Power Supplies. 1986; 445-449.

3. Yeh C-C, Manjrekar MD. A reconfigurable uninterruptible power supply system for multiple power quality applications. Twentieth Annual IEEE Applied Power Electronics Conference and Exposition. 2005 March; 1824-1830.

4. Rech C, Pinheiro H, Grundling HA, Hey HL, Pinheiro JR. Analysis and design of a repetitive predictive-PID controller for PWM inverters. IEEE 32nd Annual Power Electronics Specialists Conference. 2001; 986-991.

5. Ding X, Qian Z, Yang S, Cui B, Peng F. A PID Control Strategy for DC-link Boost Voltage in Z-source Inverter. APEC 07 - Twenty-Second Annual IEEE Applied Power Electronics Conference and Exposition. 2007; 1145-1148.

6. Tehrani K-A, Capitaine T, Barrandon L, Hamzaoui M, Rafiei SMR, Lebrun A. Current control design with a fractionalorder PID for a three-level inverter. Proceedings of the 2011 14th European Conference on Power Electronics and Applications. 2011 September.

7. Bolat ED, Ertunc HM. Implementation of Current Mode Fuzzy Tuning-PI Control of Single Phase UPS Inverter Using DSP. Knowledge-Based Intelligent Information and Engineering Systems. 2005 August; 600-607.

8. Mir SA, Zinger DS, Elbuluk ME. Fuzzy controller for inverter fed induction machines. Conference Record of the
1992 IEEE Industry Applications Society Annual Meeting. 1994 Jan.-Feb.; 30(1): 78-84.

9. Jian L, Yong K, Jian C. Fuzzy-tuning PID control of an inverter with rectifier-type nonlinear loads. Third International Power Electronics and Motion Control Conference. 2000; 381-384.

10. Ding X, Qian Z, Yang S, Cui B, Peng F. A direct DC-link boost voltage PID-like fuzzy control strategy in Z-source inverter. 2008 IEEE Power Electronics Specialists Conference. 2008 June; 405-411.

11. Thao NGM, Dat MT, Binh TC, Phuc NH. PID-fuzzy logic hybrid controller for grid-connected photovoltaic inverters. International Forum on Strategic Technology. 2010 October.,

12. Li JD, Wei SZ, Qiong W, Peng X. A Switching-inverter power controller based on fuzzy adaptive PID. Proceedings of 2011 6th International Forum on Strategic Technology. 2011 August; 695-699.

13. Chen W-L, Chiu Y-M, Li Z-C, Yen C-S, Wu D. A novel gain scheduling application for voltage-sourced inverters control based on internal model principle. 2012 IEEE International Conference on Power System Technology (POWERCON). Auckland 2012.

14. Jiao J, Hung JY, Nelms RM. Gain scheduling control strategy for a single-phase grid-connected inverter. IEEE 26th International Symposium on Industrial Electronics (ISIE). 2017 June; 723-728.

15. Yilmaz AR, Erol B, Delibaş1 A, Erkmen B. Design of gainscheduling PID controllers for Z-source inverter using iterative reduction-based heuristic algorithms. Simulation Modelling Practice and Theory 94. 2019 February: 162-176.

16. Datta A, Mukherjee D, Debbarma S, Saha H. A dsPIC based efficient single-stage grid-connected photovoltaic system. TENCON IEEE Region 10 Conference. 2014 October.

17. Ogata K. Modern Control Engineering: Pearson; 2009.

18. Arınç R. Bulanık Mantık Yöntemiyle Asenkron Motor Hız Kontrolü ve Simülasyonu. Yüksek Lisans Tezi. İstanbul: Marmara Üniversitesi Fen Bilimleri Enstitüsü; 2003.

19. Yu K-W, Hwang R-C, Hsieh J-G. Automatic ship handling of maritime search mission by using self-tuning fuzzy gain scheduling PD controller. Journal of Navigation. 1999 September; 52(3): 378-387. 